\title{
Prediction of Elastic Properties for Unidirectional Carbon Composites: Periodic Boundary Condition Approach
}

\author{
Kamarul Azhar Kamarudin ${ }^{a^{*}}$, Al Emran Ismail ${ }^{b}$ \\ Faculty of Mechanical and Manufacturing Engineering, Universiti Tun Hussein Onn Malaysia, Batu \\ Pahat, 86400 Johor, Malaysia \\ akamarula@uthm.edu.my, bemran@uthm.edu.my
}

Keyword: Mechanical Properties, Mechanical Testing, Unit Cell, Periodic Boundary Conditions

\begin{abstract}
A three-dimensional finite element model of unidirectional fibre reinforced composites has been investigated numerically using periodic boundary condition method. This method was used to predict the elastic mechanical behaviour of a unit cell of such composites. Periodic boundary condition was used due to its capability to represent a single unit cell similar to the neighbouring unit cells with continuous physical elements. It is assumed that the paired nodes displaced continuously without separating or interrupting other nodes during the deformation step. From the study, it was revealed that the elastic modulus agreed well with the experimental results, indicating that the present model could be used effectively.
\end{abstract}

\section{Introduction}

The past decades have seen rapid development in micromechanics elastic modulus prediction such as the use of energy variation principles [1], energy balance approach [2] and mechanics approach [3]. Another technique known as periodic boundary condition (PBC) method has been studied extensively by [4-6]. The early development of PBC used a 2D model to solve the problem. Li [4] developed a general packing approach that is able to accommodate unidirectional fibre of irregular cross sections. Later, Li and Wongsto [7] extended their research to 3D environment involving irregular geometries and local imperfection within composites. Xia et al. [5] presented unified periodic displacement boundary conditions in displacement-based FEM, which is applied into 2D multi-shaped models. Later in 2007, Tyrus et al. [6] demonstrated the study on 2D triangular and square shapes which focused on the effectiveness of computational speed.

In many publications, the analysis using periodic boundary condition is modelled to a single unit cell $[7,8]$ while few using multilayer composite $[9,10]$. This paper attempts to reveal the elastic properties results from $\mathrm{PBC}$ in comparison with the experimental study, as well as the elastic prediction on unidirectional micromechanics of a single unit cell. In addition, the final results try to examine the application of periodic boundary condition method in a multi-layer unidirectional and bidirectional composite in three-dimensional model analysis.

\section{Material Properties and Composite geometry}

The unidirectional laminate of epoxy resin impregnated AS4 carbon fibre was used in the study and the properties are taken from [11] as shown in Table 1.

Table 1: Properties of composite [11]

\begin{tabular}{ccc}
\hline Properties $(\mathrm{GPa})$ & Fibre AS4 & Resin \\
\hline $\mathrm{E}_{1}$ & 235 & 4.8 \\
$\mathrm{E}_{2}$ & 14 & 4.8 \\
$\mathrm{G}_{12}$ & 28 & 1.8 \\
$\mathrm{v}_{12}$ & 0.2 & 0.34 \\
$\mathrm{v}_{23}$ & 0.25 & 0.34 \\
\hline
\end{tabular}


The model of matrix and fibre in Table 1 has the dimension of $2 \times 2 \times 2$ that represents $a, b$ and $c$, with fibre fraction measurement of 0.6. By keeping the fibre length constant, the fibre diameter is 0.87 in radius. Due to expression for transverse isotropic to the material, only four different loading conditions will be determined i.e. $E_{1}, E_{2}, G_{12}$ and $G_{23}$. An element chosen for meshing purposes in ABAQUS was C3D8R.

\section{Periodic Boundary Conditions for Repeating Unit Cells (RUCs)}

Periodic methods are composed of fundamental building blocks called repeating unit cells (RUCs) which when assembled together side by side will form an infinite array. The displacement of each unit cell must be continuous, which means that the neighbouring RUC cannot be separated from each other after the deformation. In addition, traction distribution should also be similar at the opposite parallel boundaries of a RUC. This means that the element in forming macroscopic structure will produce the individual RUC, which can thus be assembled as a physically continuous body. Internal microstructure within the RUC is not important but only within the outer surface of the whole structure is considered.

To generalise the model into a cubic unit cell, subscripts 1 and 2(in Fig. 1) indicate the position before and after the translational of $x, y, z$ three-dimensional directions. Any parts of the given volume relatively have displacement of any point away from the existing cell tied with the number of cells $i, j, k=0, \pm 1, \pm 2, \pm 3 \ldots$, which is related with:

$$
\left(x_{2}, y_{2}, z_{2}\right)=\left(x_{1}+i b, y_{1}+j b, z_{1}+k b\right)
$$

and formed as:

$u_{2}-u_{1}=(i b) \bar{\varepsilon}_{x}+(j b) \bar{\gamma}_{x y}+(k b) \bar{\gamma}_{x z}$

$v_{2}-v_{1}=(j b) \bar{\varepsilon}_{y}+(k b) \bar{\gamma}_{y z}$

$w_{2}-w_{1}=(k b) \bar{\varepsilon}_{z}$

The equations established above are related with the displacement boundary condition that can be prescribed by macroscopic strains as loads, which can be treated as an independent degree of freedom to the system. With regards to the displacement of boundary conditions, positions $x, y$ and $z$ at origin $O$ are constrained from displacements in all respective directions. In addition, the $x$-axis is constrained by the rotation of the $z$-axis. By explaining mathematically at origin, $O$ :

$$
\mathrm{x}, \mathrm{y}, \mathrm{z}=0, \frac{\partial u}{\partial z}=\frac{\partial v}{\partial z}=\frac{\partial w}{\partial y}=0
$$

Concentrated forces related forms are expressed as strains rather than displacements and are applied for the key degree of freedom. It is also equivalent to the key nodal forces. Macroscopic stresses that are related to the concentrated forces are applied on this key degree of freedom. These manners are similar when considering simple energy equivalent as the work done by concentrated forces over the macroscopic strain is similar as the macroscopic stress over the macroscopic strain for the volume of unit cell. For instance, if a force in the x-direction is applied to the degree of freedom $\varepsilon_{x}$ of the unit cell, while all other degrees of freedom are free from constrains, the work done by the force is taken as:

$W=\frac{1}{2} F_{x} \varepsilon_{x}$

Meanwhile, the strain energy stored in the unit cell can be expressed in macroscopic stress and strains as: 
$E=\frac{1}{2} \int_{V} \sigma_{x} \varepsilon_{x} d V=\frac{1}{2} V \sigma_{x} \varepsilon_{x}$

where $V$ is the volume of unit cell and work done $\mathrm{W}$ is equal to the strain energy. Equalizing both strain energy and work done on the unit cell gives simple stress relations obtained as:

$$
\sigma_{x}=\frac{F_{x}}{V} ; \quad \sigma_{y}=\frac{F_{y}}{V} ; \quad \sigma_{z}=\frac{F_{z}}{V} ;
$$

where $x, y$, and $z$ are the directions of forces acting on the volume $V$ of the unit cell. To make it simpler, it can be said that the magnitude of the concentrated load (F) is equal to the volume of the unit cell $(V)$ times the stress it will generate macroscopically. In this study, the macroscopic stress is taken as $1 \mathrm{MPa}$. The effective material properties on the 'key nodes' for Young's modulus parallel to the fibre direction $E_{x}, E_{y}, E_{z}, G_{y z}, G_{x z}$ and $G_{x y}$ and can be obtained as:

\begin{tabular}{|c|c|c|c|c|}
\hline Direction & Apply & Displacement formed in & To determine & \\
\hline $\mathrm{x}$ & $\sigma_{x}$ only & $\varepsilon_{x}$ & $E_{x}=\frac{\sigma_{x}}{\varepsilon_{x}}$ & \multirow{6}{*}{ (Eq. 7 ) } \\
\hline $\mathrm{y}$ & $\sigma_{y}$ only & $\varepsilon_{y}$ & $E_{y}=\frac{\sigma_{y}}{\varepsilon_{y}}$ & \\
\hline $\mathrm{z}$ & $\sigma_{z}$ only & $\varepsilon_{z}$ & $E_{z}=\frac{\sigma_{z}}{\varepsilon_{z}}$ & \\
\hline yz & $\tau_{y z}$ only & $\gamma_{y z}$ & $G_{y z}=\frac{\tau_{y z}}{\gamma_{y z}}$ & \\
\hline zX & $\tau_{z x}$ only & $\gamma_{z x}$ & $G_{z x}=\frac{\tau_{z x}}{\gamma_{z x}}$ & \\
\hline xy & $\tau_{x y}$ only & $\gamma_{x y}$ & $G_{x y}=\frac{\tau_{x y}}{\gamma_{x y}}$ & \\
\hline
\end{tabular}

\section{Results and Discussions}

Unidirectional Composite using PBC approach. Fig. 1 illustrates stress distribution of periodic boundary condition with regards to elastic constant. This scenario of boundary condition for PBC showed a continuous deformation along its boundaries. The simulation model also showed a convincing method to be used for different model geometry shapes.

\begin{tabular}{|l|l|l|l|l|}
\hline & $E_{1}$ & $E_{2}$ & $G_{12}$ & $G_{23}$ \\
\hline & & & & \\
\end{tabular}

Fig. 1: Stress distribution using periodic boundary condition method. 
Table 2: Comparison of elastic constants for CFRP

\begin{tabular}{cccc|c|c|c|c}
\hline \multicolumn{4}{c|}{ Unidirectional Composite } & \multicolumn{3}{c}{ Bidirectional Composite } \\
\hline $\begin{array}{c}\text { Elastic } \\
\begin{array}{c}\text { Constants } \\
(\mathrm{GPa})\end{array}\end{array}$ & {$[12]$} & {$[13]$} & {$[14]$} & Present & $\begin{array}{c}\text { Elastic } \\
\text { Constants } \\
(\mathrm{GPa})\end{array}$ & $\begin{array}{c}{[15]} \\
\mathrm{V}_{\mathrm{f}}=60 \%\end{array}$ & Present \\
\hline$E_{1}$ & 142.6 & 142 & 139 & 143.94 & $E_{1}=E_{3}$ & $65.9 / 61.8$ & 76.14 \\
$E_{2}$ & 9.60 & 10.30 & 9.85 & 9.71 & $E_{2}$ & 11.3 & 10.42 \\
$G_{12}$ & 6.00 & 7.60 & 5.25 & 6.16 & $G_{12}=G_{32}$ & $6.98 / 7.14$ & 4.96 \\
$G_{23}$ & 3.10 & 3.8 & - & 4.48 & $G_{13}$ & 15.79 & 11.68 \\
$v_{12}$ & 0.25 & - & 0.3 & 0.25 & $v_{12}$ & 0.46 & 0.35 \\
$v_{23}$ & 0.35 & - & - & 0.35 & $v_{13}$ & 0.052 & 0.031 \\
\hline
\end{tabular}

Bidirectional Composite using PBC approach. To extend the technique on another model, a unit cell of bidirectional fibrous composite was developed. Fig. 2 shows a RUC of fibre at cross angle of $0^{\circ}$ and $90^{\circ}$ respectively in its $x z$-plane.

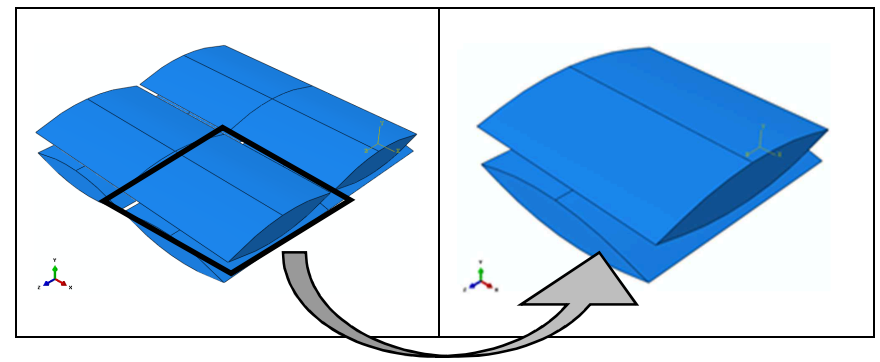

Fig. 2: Bidirectional elliptical RUC fibre selection (left) full size (right) quarter size.

\begin{tabular}{|c|c|c|c|}
\hline$\sigma_{x}$ & $\sigma_{y}$ & $\tau_{x y}$ & $\tau_{y z}$ \\
\hline & & & \\
\hline & & & \\
\hline
\end{tabular}

Fig. 3: Stress distribution for bidirectional composite.

Using periodic method, the model was analysed and performed as illustrated in Fig. 3. $\sigma_{1}$ and $\sigma_{3}$ produced the same elastic value due to the symmetric fibre orientation while $\mathrm{E}_{2}$ will approximately be $10 \%$ of the $E_{1}$ and $E_{3}$ values. The results of the $60 \%$ fibre volume bidirectional CFRP analysis are shown in Table 2. There are numbers of similarities of the elastic properties which resulted from the PBC methods against experimental result by other researchers. The accuracy of the PBC method for elastic constants prediction against the existing results is tested.

\section{Conclusion}

The focus of this paper is to compare elastic properties for reinforced composite fibre using periodic boundary condition method. The method was introduced on micromechanical unit cells with sets of boundary conditions applied. The translation of displacement along the boundary condition at the surrounding unit cell was symmetrical, including translations, reflections and rotations. Good agreement was obtained between the predicted and test results for all models. 


\section{Acknowledgement}

The author would like to thank Universiti Tun Hussein Onn Malaysia and the Ministry of Higher Education Malaysia for their financial support.

\section{References}

[1] Hashin Z and Rosen BW (1964), The elastic moduli of fiber-reinforced materials. ASME J Appl Mech. 31: 223-32

[2] Whitney JM and Riley MB (1966), Elastic properties of fiber reinforced composite materials. AIAA J, 4:1537-42.

[3] Sun CT and Chen JL (1990), A micromechanical model for plastic behaviour of fibrous composites, Comp Sci Technol. $40: 115-29$.

[4] Li S (2001), General unit cells for micromechanical analyses of unidirectional composites. Composites Part A: Applied Science and Manufacturing 32(6): 815-826.

[5] Z, Zhou C, Yong Q and Wang X (2006), On selection of repeated unit cell model and application of unified periodic boundary conditions in micro-mechanical analysis of composites, Int. Journal of Solids and Structures, 43(2): 266-278.

[6] Tyrus JM, Gosz M and DeSantiago E (2007), A local finite element implementation for imposing periodic boundary conditions on composite micromechanical models. Int. Journal of Solids and Structures 44(9): 2972-2989.

[7] Li S and Wongsto A (2004), Unit cells for micromechanical analyses of particle-reinforced composites, Mechanics of Materials, 36(7): 543-572.

[8] Abolfathi N, Naik A, Karami G and Ulven C (2008), A micromechanical characterisation of angular bidirectional fibrous composites. Computational Material Science 43:1193-1206.

[9] Drago A and Pindera M, (2007), Micro-macromechanical analysis of heterogeneous materials: Macroscopically homogeneous vs periodic microstructures, Composites Science and Technology, 67(6): 1243-1263.

[10] Ohno N, Wu X and Matsuda T (2000), Homogenized properties of elastic-viscoplastic composites with periodic internal structures, International Journal of Mechanical Sciences, 42(8): 1519-1536.

[11] King TR, Blackketter DM, Walrath DE and Adams DF (1992), Micromechanics Prediction of the Shear Strength of Carbon Fiber/Epoxy Matrix Composites: The Influence of the Matrix and Interface Strengths, Journal of Composite Materials, 26: 558-573.

[12] Sun CT and Vaidya RS (1996), Prediction of composite properties from a representative volume element. Composites Science and Technology, 56(2): 171-179.

[13] Lee JW and Daniel IM (1990), Progressive transverse cracking of cross ply composite laminates, Journal of Composite Material, 24: 1225-43.

[14] Sun CT and Zhou SG (1988), Failure of quasi-isotropic composite laminates with free edges, Journal of Reinfastructure Plastic Composite, 7: 515-57.

[15] Kim BC, Park DC, Kim BJ and Lee DG (2010), Through-thickness compressive strength of a carbon/epoxy composite laminate, Composite Structure, 92(2): 480-487. 